\title{
EDUCAÇÃO POSTURAL EM CRIANÇAS EM IDADE ESCOLAR: REVISÃO INTEGRATIVA DA LITERATURA
}

EDUCACIÓN POSTURAL EN NIÑOS EN EDAD ESCOLAR: REVISIÓN DE LA LITERATURA INTEGRADORA

\author{
POSTURAL EDUCATION IN SCHOOL AGED CHILDREN: INTEGRATIVE LITERATURE REVIEW
}

DOI 10.33194/rper.2020.v3.n2.9.5812 | Submetido 09/10/2020 | Aprovado 15/12/2020

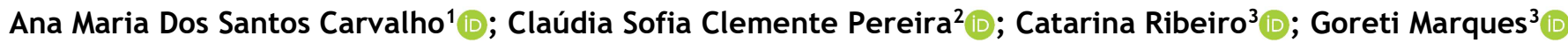 \\ 1 - Centro Hospitalar de Vila Nova de Gaia/Espinho e Escola Superior de Saúde de Santa Maria; \\ 2 - ASAS - Gabinete de Psicologia e Terapias complementares; 3 - Escola Superior de Saúde de Santa Maria
}

\section{RESUMO}

Enquadramento: A educação postural é essencial para promover uma postura corporal adequada e prevenir a adoção de posturas incorretas, das quais podem resultar dor e lesões musculosqueléticas ${ }^{(1,2,3,4,5)}$. A implementação de programas a nível escolar tem uma forte influência na saúde e na qualidade de vida das crianças ${ }^{(6,7)}$.

Objetivo: Analisar a evidência existente relativa aos programas de educação postural implementados a nível escolar.

Metodologia: Através da realização de uma revisão integrativa da literatura, utilizou-se a metodologia PEO (participantes, exposição e resultados). A colheita de dados foi realizada em abril de 2020, nas bases de dados CINAHL e PubMed.

Resultados: A análise dos artigos incluídos permitiu identificar que os programas de educação postural apresentam benefícios ao nível do conhecimento, dos parâmetros posturais estáticos e dinâmicos, da redução do peso das mochilas, do nível de dor musculosquelética e do desalinhamento do ombro.

Conclusão: A evidência demonstra que a implementação de programas de educação postural, a nível escolar, tem resultados positivos na promoção da saúde das crianças.

Palavras-chave: postura; serviços de saúde escolar; criança; enfermagem de reabilitação

\section{RESUMEN}

Antecedentes: La educación postural es esencial para promover una postura corporal adecuada y evitar la adopción de posturas incorrectas, que pueden provocar dolor y lesiones musculoesqueléticas ${ }^{(1,2,3,4,5)}$. La implementación de programas escolares tiene una fuerte influencia en la salud y la calidad de vida de los niños ${ }^{(6,7)}$.

Objetivo: Analizar la evidencia existente sobre los programas de educación postural implementados a nivel escolar.

Metodología: Mediante una revisión bibliográfica integradora, se utilizó la metodología PEO (participantes, exposición y resultados). La recopilación de datos se llevó a cabo en abril de 2020, en las bases de datos CINAHL y PubMed.

Resultados: El análisis de los artículos incluidos nos permitió identificar que los programas de educación postural presentan beneficios en términos de conocimiento, parámetros posturales estáticos y dinámicos, reducción de peso en la mochila, nivel de dolor musculoesquelético y desalineación del hombro.

Conclusión: La evidencia muestra que la implementación de programas de educación postural, a nivel escolar, tiene resultados positivos en la promoción de la salud de los niños.

Palabras clave: postura; servicios de salud escolar; niño; enfermería de rehabilitación

\section{ABSTRACT}

Background: Postural education is essential to promote proper body posture and prevent the adoption of incorrect postures, which can result in pain and musculoskeletal damage $(1,2,3,4,5)$. The implementation of school-level programs has a strong influence on children's health and quality of life ${ }^{(6,7)}$.

Objective: To analyze the existing evidence concerning postural education programs implemented at school level.

Methodology: Through an integrative literature review, the PEO methodology (participants, exposure and results) was used. The data collection was conducted in April 2020 in the CINAHL and PubMed databases.

Results: The analysis of the articles included allowed us to identify that postural education programs have benefits in terms of knowledge, static and dynamic postural parameters, backpack weight reduction, musculoskeletal pain level and shoulder misalignment. 
Conclusion: The evidence shows that the implementation of postural education programs, at school level, has positive results in promoting children's health.

Keywords: posture; school health services; child; rehabilitation nursing

\section{INTRODUÇÃO}

A postura corporal pode ser definida como a posição ou atitude do corpo, o arranjo relativo das partes corporais para uma atividade específica, tornando-se uma forma característica de uma pessoa sustentar o seu corpo ${ }^{(2)}$. Assim, segundo os mesmos autores, uma boa postura reflete a capacidade dos ligamentos, cápsulas e tónus muscular de suporte do corpo ereto, permitindo a sua permanência numa determinada posição, por períodos prolongados, sem desconforto e com baixo consumo energético.

Em contrapartida, uma má postura origina stress total sobre os elementos do corpo, especialmente sobre a coluna vertebral, podendo gerar desconfortos, dores ou incapacidades funcionais ${ }^{(3)}$.

De acordo com Milbradt \& Pranke (8), as dorsalgias, que podem afetar a região cervical, dorsal e lombar da coluna vertebral, as alterações do padrão cinemático da marcha e do equilíbrio, a dificuldade de sustentação e de mobilidade do sistema musculosquelético, a escoliose, a hipercifose torácica, a hiperlordose lombar e as patologias osteoarticulares e musculotendinosas são algumas das consequências, inumeradas em diversos estudos, de uma postura corporal desadequada e que, por sua vez, condicionam a qualidade de vida do ser humano ${ }^{(2,4)}$.

Os hábitos posturais vão sendo adquiridos ao longo da vida sob influência de comportamentos e fatores socioeconómicos ${ }^{(9,10)}$, contudo, sabe-se hoje, que é na infância que se encontram as maiores variações posturais, fruto dos vários ajustes, adaptações e mudanças corporais e psicossociais que marcam esta fase de desenvolvimento ${ }^{(2)}$.

Estudos recentes apontam ainda que, no período escolar, o sistema músculo-esquelético encontra- se em processo de crescimento e fortemente vulnerável a fatores externos que levam muitas vezes a posturas incorretas das crianças e jovens ${ }^{(11)}$. 0 uso de mobiliário inadequado, a utilização regular de mochilas escolares, o transporte de cargas excessivas nas mochilas, o uso inapropriado do corpo nas atividades de estudos, o sedentarismo e a obesidade parecem estar fortemente associados à aquisição de maus hábitos posturais na infância ${ }^{(3,11,12)}$.

Por outro lado, a idade escolar parece, também, ser a mais propícia para a mudança, uma vez que, através de estratégias lúdico-pedagógicas, desenvolvidas por equipas de saúde escolar, é possível ensinar novos hábitos posturais às crianças, promovendo assim o seu bem estar e qualidade de vida no futuro ${ }^{(13,14,15)}$.

A promoção da educação postural a crianças deve, por isso, ser um objetivo prioritário ao nível da intervenção na área da Saúde Escolar, tendo os enfermeiros especialistas em enfermagem de reabilitação um papel fulcral, como profissionais de saúde, no meio escolar no âmbito da prevenção primária em saúde ${ }^{(16)}$.
Segundo Santos (7), a promoção da saúde músculoesquelética e educação postural na escola tem vindo a demonstrar uma importância crescente, tornando-se uma das metas da OMS para o ano 2020. Ainda de acordo com a mesma autora (7), a utilização de programas de educação revela vantagens significativas, por um lado porque abrange uma grande parte da população e, por outro, porque possibilita o acompanhamento da mesma por longos períodos de tempo, permitindo, assim, obter uma avaliação mais específica da eficácia dos mesmos.

De um modo geral, os programas de educação postural permitem promover melhorias no conhecimento e no comportamento dos alunos em relação aos seus hábitos e à sua saúde, sensibilizando para uma correta postura corporal (1). Por outro lado, os programas educacionais facilitam a implementação de estratégias específicas no âmbito da ergonomia escolar, na medida em que através dos mesmos se procura adequar os espaços da escola (como a utilização de cadeiras confortáveis, adequação do peso das mochilas, entre outros), de modo a potenciar o bem-estar físico e até o sucesso escolar, contribuindo assim para o desenvolvimento saudável das crianças ${ }^{(6)}$.

Tendo em conta a pertinência deste tema, com este estudo pretende-se reunir e analisar o conhecimento existente sobre os programas de educação postural que são implementados a nível escolar.

\section{MATERIAL E MÉTODOS}

A necessidade de reunir o conhecimento existente sobre os programas de educação postural que são implementados ao nível da saúde escolar, justificou a realização desta revisão integrativa, a partir da seguinte questão de investigação: Quais os programas de educação que influenciam a postura da criança em idade escolar?

Para esta revisão, foi utilizada a estratégia PEO, em que a população $(P)$ a ser estudada são as crianças dos 6-12 anos de idade, com exposição $(E)$ a programas de saúde de educação postural, a nível escolar e os resultados ou outcome (0) são a melhoria da postura corporal. A colheita dos dados ocorreu durante o mês de abril de 2020 nas bases de dados CINAHL Plus with Full Text a partir da plataforma EBSCOhost e PubMed Central. Foram utilizados os seguintes descritores para pesquisa nas bases de dados: Child, schoolchildren, School Health, School Health Services, Health Promotion, School Health Promotion, Education, Health Education, Program Implementation, Posture, em combinação com AND e OR.

Foram incluídos artigos publicados entre 2015 e 2020 nas línguas inglês, português e espanhol, disponíveis em texto integral. Foram, assim, identificados 141 artigos através da pesquisa nas bases de dados, conforme se pode verificar na figura 1 : 


\section{RESULTADOS}

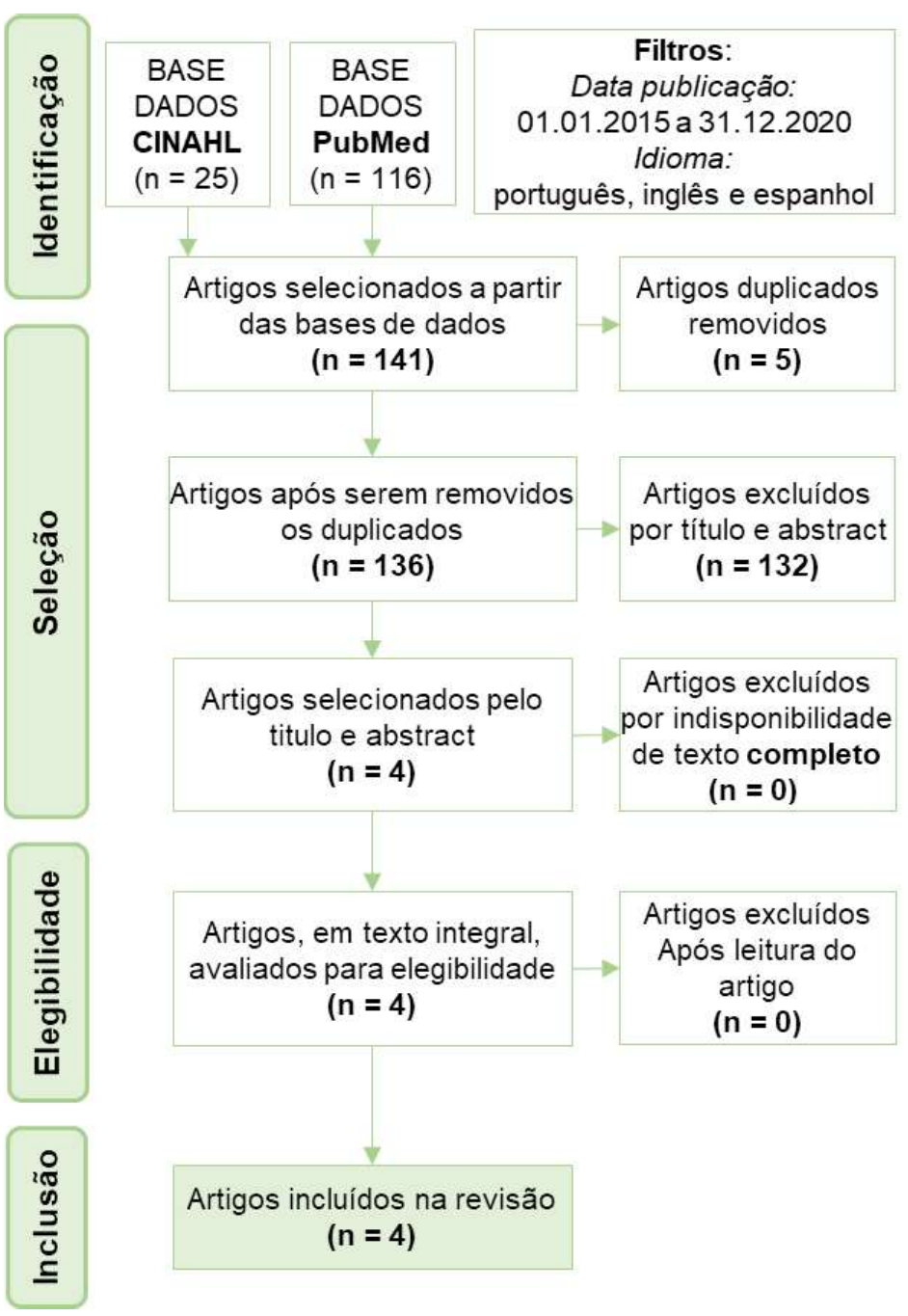

Figura 1. Diagrama do processo de seleção dos estudos

Provenientes da base de dados CINAHL foram identificados 25 artigos e 116 artigos provenientes da base de dados PubMed. Seguidamente, dos 141 artigos identificados, foram removidos cinco artigos por estarem duplicados, de seguida foi realizada uma seleção dos artigos a partir da leitura do título e do resumo de cada artigo, dos quais foram extraídos 132 artigos, por não responderem aos critérios da pergunta de investigação desta revisão. Foram selecionados quatro artigos por leitura de título e resumo que se verificaram serem elegíveis por estarem disponíveis em full text e responderem á questão de investigação. Após leitura de texto integral dos quatro artigos, foram incluídos para revisão os mesmos uma vez que cumpriram todos os critérios de inclusão.

Os artigos selecionados foram analisados de forma criteriosa e foram extraídos dados que possibilitaram a compilação, numa tabela, com a seguinte informação: autores do artigo, ano de publicação, tipo de estudo, intervenção, conteúdo do programa de educação e resultados.
Após análise e leitura dos quatro artigos verificou-se que um dos artigos foi desenvolvido na Polónia, outro na Alemanha e dois artigos no Brasil. O Brasil é o país que mais se destaca pela quantidade de estudos realizados sobre a temática da postura em crianças em idade escolar (17).

Dos artigos analisados, três foram publicados no idioma inglês e um foi publicado em português. Quanto á data de publicação, um dos artigos foi publicado no ano de 2016, um em 2017, um em 2018 e outro em 2019.

0 primeiro artigo analisado, tem como autores Brzek e Plinta ${ }^{(18)}$, que em 2016, realizaram um estudo com uma amostra constituída por crianças sem alterações posturais diagnosticadas, na Polónia, divididas em grupo de intervenção e grupo de controle. 0 grupo de intervenção foi composto por 144 participantes com idades entre os sete e os nove anos, de uma escola onde o programa "I take care of my spine" estava implementado. 0 grupo de controle foi composto por 222 crianças com idades entre os sete e os nove anos, que frequentavam escolas, onde o programa não tinha sido implementado. 0 objetivo deste estudo foi avaliar a postura corporal das crianças que frequentaram o programa "I take care of my spine", em comparação com outras crianças, que não frequentaram este programa. A intervenção consistiu na participação de ambos os grupos em aulas de educação física de 45 minutos cada, três a quatros vezes por semana. Todos os participantes foram submetidos a uma avaliação inicial, nas mesmas condições, antes do programa ser lançado com recurso a instrumentos (linha de prumo, escoliómetro da Pedihealth Oy, Finlândia e um inclinómetro digital - SAUNDERS TMX-127) e a testes de avaliação postural, seguindo-se um processo de exame detalhado. Foi realizada uma segunda avaliação passados nove a dez meses de participação completa no programa e depois de um ano de observação do grupo de controle. De forma complementar, as crianças responderam a questionários, por duas vezes, relativos a posturas da vida quotidiana desempenhadas durante a aprendizagem e durante as brincadeiras ou atividades de tempos livres. 0 peso das mochilas foi medido entre o nono e o decimo mês de participação.

Como resultados, verificou-se uma melhoria significativa dos parâmetros posturais no grupo de intervenção que frequentou o programa "I take care of my spine" e um agravamento dos mesmos parâmetros no grupo de controle, que apenas foi observado. No grupo que frequentou o programa postural, o peso das mochilas diminuiu significativamente.

0 artigo realizado por Santos, Sedrez, Candotti e Vieira ${ }^{(15)}$, em 2017, apresenta uma amostra constituída por 38 participantes com idades entre os oito e os doze anos, do terceiro ano do ensino fundamental, do Brasil. Tem como objetivo avaliar os efeitos a curto e médio prazo da implementação de um programa de educação postural em alunos do ensino fundamental.

Todos os alunos frequentaram o programa de educação postural constituído por dez momentos: um para o préteste, oito para os conteúdos teórico-práticos (aulas de 90 minutos, uma vez por semana) e um para o pós- 
teste. 0 reforço da aprendizagem foi feito três meses após o programa, com aulas de 90 minutos quinzenalmente, perfazendo quatro momentos, onde se fez revisão de conteúdos. Uma semana depois do quarto momento de revisão, foi feito o follow-up dos cinco meses.

Os participantes foram avaliados através de questionário (avaliação de conhecimento teórico acerca da coluna vertebral e da postura corporal) e de um circuito de filmagem (avaliação da postura dinâmica através do Layout for assessing the dynamic posture LADy) no pós-teste e no follow-up aos cinco meses; no pré-teste foi avaliada apenas a postura dinâmica.

Como resultados, verificou-se que os participantes mantiveram um nível de conhecimento teórico semelhante sobre questões anatómicas da coluna vertebral e hábitos posturais no pós-teste e teste de follow-up. Apresentaram na avaliação da postura dinâmica valores com diferença significativa no score final do LADy entre o pré-teste e o pós-teste e também entre o pré-teste e o follow-up. Dos itens avaliados no LADy apenas um dos cinco itens, não apresentou diferença significativa entre os momentos de avaliação.

0 artigo realizado por Dullien, Grifka e Jansen ${ }^{(19)}$, em 2018, incluiu uma amostra de 176 participantes com idades dos 10 anos 12 anos, a frequentar o quinto ano de escolaridade de duas escolas alemãs, perfazendo quatro turmas de cada escola. Foram divididos, de forma aleatória, em grupo de intervenção e grupo de controle. Este estudo, teve como objetivo avaliar se um programa de educação postural conduzido por professores, poderia melhorar as dores na região dorsolombar dos alunos, as capacidades motoras, o comportamento e o conhecimento. Durante 10 meses, um professor deu cinco aulas: melhorou conhecimento dos alunos, fez treino de consciência postural e fez exercícios de reforço muscular dos músculos dorsais e abdominais. A avaliação do pré-este e pós-teste incluiu um exame ortopédico clínico, um questionário de saúde, um teste motor, uma prova de avaliação do comportamento da região dorsal e um teste de conhecimentos, que foi realizado a ambos os grupos. Foi realizada uma avaliação intercalar de conhecimentos e uma prova de avaliação do comportamento da região dorsal, depois de terminar as cinco aulas do programa, apenas ao grupo de intervenção.

Os resultados mostraram que a taxa de dor não reduziu. De referir que os conhecimentos sobre os cuidados a ter com a região dorsal e os aspetos do comportamento favorável à região dorso-lombar melhoraram significativamente, mas persiste o problema das longas horas na posição sentada e da utilização de mochilas pesadas. Não se registou qualquer melhoria significativa do comportamento, no que respeita à resistência dos músculos do tronco.

O último artigo analisado foi realizado por Batistão, Carnaz, Moreira e Sato(20), em 2019, e incluiu uma amostra de 171 participantes a frequentar o ensino fundamental, de três escolas públicas do Brasil. Estes, foram posteriormente divididos em grupo de intervenção e grupo de controle. Teve como objetivo avaliar os efeitos de um programa de exercícios de alongamento e fortalecimento muscular, implementado em ambiente escolar, na postura, mobilidade de tronco e dor músculo-esquelética.

O grupo de intervenção participou no programa, implementado por um fisioterapeuta, que promoveu exercícios de alongamento e fortalecimento muscular em grupos de dez participantes, durante duas vezes por semana, em que cada sessão teve a duração de 50 minutos, durante oito semanas. Os dados demográficos foram recolhidos através de formulário standard, foi avaliada a presença de dor por relato do próprio através de um questionário, foi avaliada a postura (antes e depois da intervenção) por um fisioterapeuta, com recurso a uma plataforma giratória, uma câmara digital fotográfica, um tripé e uma linha de prumo; foi, ainda, utilizado um software de avaliação postural. A mobilidade do tronco foi avaliada através de fotogrametria.

Os resultados demonstram uma redução do nível de dor musculosquelética e do desalinhamento do ombro, no grupo de intervenção. No entanto, estes não foram significativos para outros desvios posturais, como a postura da cabeça e do tronco.

\section{DISCUSSÃO}

Verificou-se alguma homogeneidade relativamente ao tipo de estudo, bem como às características da população. No entanto, existe muita diversidade no que se refere ao tipo de intervenção, conteúdo do programa de educação postural e período de implementação do programa.

No estudo de Brzek \& Plinta (18) o programa implementado foi "I take care of my spine" dirigido a crianças, pais e professores e foi executado por um período de seis anos em instituições escolares (20102015), integrando o programa escolar. O conteúdo deste programa de educação postural tem duas componentes, uma de ensino e outra de exercício físico. Durante as aulas às crianças foram abordados temas sobre: a estrutura e função da coluna vertebral, padrões de movimento adequados e inadequados, esclarecimentos sobre as causas das perturbações posturais e como estas podem afetar as suas capacidades físicas enquanto adultos. As atividades realizadas neste programa, foram enriquecidas com variados meios audiovisuais avançados, que auxiliaram a desenvolver um sentido de responsabilidade para si próprios e para os outros. Para as crianças envolvidas neste programa, a realização de ginástica entre as aulas passou a ser uma parte fixa do dia escolar. Os pais foram instruídos sobre como criar um local de trabalho ergonómico para os seus filhos e como controlar constantemente uma posição corporal ideal. No programa foi realizado treino comportamental e abordada a questão do peso da mochila escolar.

Num outro estudo de Santos et al. (15) dirigido a crianças, o programa abrangia aulas teórico-práticas envolvendo o estudo das estruturas, curvaturas e funções da coluna vertebral, atividades de vida diárias, tais como: permanecer em pé e caminhar, carregar a 
mochila, segurar objetos do solo, sentar, mastigar e deitar. Este programa não incluiu uma componente de exercício físico, apenas de ensino. Os conteúdos foram lecionados em contexto de sala de aulas, com a duração de 90 minutos, uma vez por semana, durante oito semanas. O reforço da aprendizagem foi feito três meses após o programa com aulas de 90 minutos quinzenalmente, em quatro momentos distintos, onde foram revistos os conteúdos abordados. Uma semana após o quarto momento de contacto, foi feito o followup dos cinco meses de programa.

Dullien et al. (19) realizaram um programa dirigido a crianças, no qual um professor liderou cinco aulas onde melhorou o conhecimento dos seus alunos, através do treino de consciência postural e exercícios de reforço muscular dos músculos dorsais e abdominais, durante um período de 10 meses. Este programa demonstrou ser bastante abrangente, uma vez que envolveu a componente de ensino, componente de treino de consciência corporal e a componente de exercício físico. Incluiu promoção de conhecimentos anatómicos da coluna vertebral, boa e má postura enquanto sentado, hábitos saudáveis de mochila, elevação saudável e transporte da mochila, desportos promotores de boa postura e nutrição. 0 treino de consciência postural foi realizado com recurso a cartazes mostrando às crianças alternativas para a posição sentada de forma a promover dinamismo sentado; foram realizados exercícios de fortalecimento do tronco e exercícios de mobilização/alongamento para melhorar tensão e encurtamento muscular. Estes exercícios foram realizados com cada aluno sentado na sua secretária. Foram, ainda, realizados exercícios para os músculos dorsais e abdominais no início de cada aula de educação física de forma a reduzir o desequilíbrio dos músculos do tronco; incluiu exercícios estáticos realizados em series de três, mantendo a posição durante 15-20 segundos e exercícios dinâmicos realizados entre 15-20 repetições- prancha, abdominais, ponte, flexão dos músculos das costas e exercícios com bola.

O programa de Batistão et al. ${ }^{(20)}$ dirigido a crianças é composto por exercícios de alongamento e exercícios de fortalecimento muscular, realizados duas vezes por semana, cujas sessões tem uma duração de 50 minutos cada, durante oito semanas. Este programa, apresenta assim, apenas a componente de exercício físico, contrastando com os restantes estudos supramencionados que apresentam também a componente de ensino. Foi explicado aos participantes a importância e o objetivo de cada exercício para assegurar a sua correta execução e foi incluído no programa o tratamento de várias alterações posturais. Os exercícios de alongamento foram dirigidos aos músculos rotadores do pescoço, flexores laterais do pescoço, elevador da escápula, trapézios superiores, eretores espinhosos, peitorais maiores e menores, romboides, flexores laterais da coluna vertebral, rotadores de coluna, piriformes, tendões, quadríceps e adutores e abdutores da anca. Os exercícios de reforço muscular foram dirigidos aos músculos da coluna cervical, músculos flexores profundos, estabilizadores da articulação gleno-umeral e escápula, abdominais, extensores da coluna vertebral e extensores da anca.
Em cada sessão de treino de exercício realizava-se primeiro o aquecimento com 10 minutos de exercícios de componente aeróbica, seguido de exercícios de fortalecimento muscular com 20-25 minutos de exercícios concêntricos em três sets de 10 repetições e exercícios isométricos de 3 sets de 5 segundos e por fim os exercícios de alongamento com 15-20 minutos de 3 sets de 30 segundos.

Após análise e apresentação destes quatro programas é possível constatar que os mesmos diferem relativamente à duração da implementação do programa que é de oito semanas para dois estudos ${ }^{(15,21)}$, de 10 meses para um dos estudos ${ }^{(19)}$ e de seis anos para outro ${ }^{(18)}$.

De salientar que apenas um único estudo dirige a intervenção do programa às crianças, pais e professores (18), enquanto os restantes estudos dirigem a sua intervenção somente às crianças ${ }^{(15,20,21)}$.

Quanto aos resultados obtidos está descrita uma melhoria significativa de conhecimentos, no estudo de Dullien et al. ${ }^{(19)}$, entre o pré-teste e o pós-teste sobre os cuidados a ter e aspetos do comportamento favorável, no grupo que cumpriu o programa. No estudo de Santos et al. (15) é relatado um nível de conhecimento de $54 \%$ sobre anatomia da coluna vertebral e de $88 \%$ sobre hábitos posturais num dos estudos, verificando-se que houve manutenção do conhecimento teórico, uma vez que mantiveram nível de conhecimentos semelhante no pós-teste e follow up. Tendo em conta que este estudo utilizou um programa apenas com a componente do ensino, a vertente do ensino demonstrou ser relevante, verificando-se efetividade da aprendizagem através da manutenção do conhecimento em follow up. Nos restantes estudos não foi avaliado o conhecimento.

Brzek \& Plinta (18) descrevem uma melhoria significativa dos parâmetros posturais nos participantes que frequentaram o programa "I take care of my spine". No estudo de Santos et al. (15) verificou-se uma melhoria significativa na avaliação da postura dinâmica no score final do LADy (Layout for assessing the dynamic posture) entre o pré-teste e o pós-teste e também entre o pré-teste e o follow-up; apenas um dos cinco itens avaliados na postura dinâmica, não apresentou diferença significativa entre os momentos de avaliação. Batistão et al. (20) descrevem, como resultado, uma redução do desalinhamento do ombro no grupo que frequentou o programa, apesar de os mesmos resultados não terem sido significativos para outros desvios como a postura da cabeça e do tronco.

Brzek \& Plinta ${ }^{(18)}$, reportam, ainda, uma redução significativa do peso das mochilas escolares, em contraste com um outro estudo de Dullien et al. ${ }^{(19)}$ que referem que se mantém o problema da utilização de mochilas pesadas.

Foi descrita uma redução do nível de dor no estudo de Batistão et al. (20), implementado durante oito semanas e apenas com componente de exercício, no entanto, no estudo de Dullien et al. (19) descrevem que não se verificou redução da taxa de dor, com período de implementação de 10 meses e com componente de ensino, treino de consciência postural e componente de 
exercício. Neste estudo reportam que no final do programa, persiste o problema das longas horas na posição sentada das crianças, o que pode justificar os resultados obtidos que mostram que a dor não reduziu, juntamente com a utilização de mochilas pesadas.

Apesar de todos os programas de educação aqui analisados seguirem abordagens diferentes, todos eles demonstram nos seus resultados apresentarem benefícios para as crianças. Os benefícios descritos nos estudos analisados envolvem a melhoria do conhecimento teórico sobre questões anatómicas da coluna vertebral e hábitos posturais, melhoria do conhecimento sobre cuidados a ter e sobre comportamento favorável, melhoria dos parâmetros posturais, melhoria da postura dinâmica, redução do peso das mochilas, redução do nível de dor musculosquelética e redução do desalinhamento do ombro. Costa et al. ${ }^{(5)}$ corrobora, nos seus estudos, os resultados encontrados verificando que os programas de saúde escolar obtêm resultados positivos, conseguindo-se prevenir lesões futuras.

Um estudo realizado por Milbradt (8) evidencia que a educação sobre posturas adequadas e o incentivo à realização de atividades de forma correta podem prevenir distúrbios posturais na juventude, prevenindo, a longo prazo, problemas decorrentes da postura como a dor lombar na idade adulta. Salientam a importância da implementação de medidas como a redução do peso das mochilas, a realização de alongamentos diários e a importância do exercício físico em ambiente escolar.

Estes resultados são concordantes com os de Costa et al. ${ }^{(5)}$, pelo que é possível concluir que a implementação de programas de educação postural nas escolas é benéfica para as crianças.

A educação postural está contemplada no PNSE 2015, com o objetivo de promover a saúde em meio escolar, apresentando-se como um objetivo das políticas nacionais de saúde ${ }^{(21)}$. A saúde escolar é direcionada a crianças e jovens cuja faixa etária é recomendado o investimento na promoção da saúde ${ }^{(22)}$. Para tal, as equipas de saúde escolar têm um papel relevante, onde o enfermeiro de reabilitação contribui de forma importante ao integrar programas de saúde escolar. Com uma abordagem centrada na família e no ciclo de vida, os enfermeiros especialistas em enfermagem de reabilitação procuram obter ganhos em saúde da população ${ }^{(4)}$.

Considerando que a postura corporal sofre influências dos hábitos posturais, dos hábitos comportamentais e dos hábitos socioeconómicos ${ }^{(8,9)}$, a educação postural é essencial para promover saúde postural através dos programas de educação em saúde escolar e desta forma, prevenir a adoção de posturas incorretas e consequentemente prevenir lesões futuras ${ }^{(5)}$.

\section{CONCLUSÃO}

Verifica-se a existência de alguma diversidade relativamente aos programas de educação postural, implementados a nível escolar nos últimos anos, no que concerne ao conteúdo do programa e à sua implementação. No entanto, todos eles descrevem benefícios ao nível do conhecimento, dos parâmetros posturais estáticos e dinâmicos, da redução do peso das mochilas, do nível de dor musculosquelética e do desalinhamento do ombro.

De salientar a relevância da vertente do ensino, verificando-se uma efetividade da aprendizagem através da manutenção do conhecimento em follow-up.

A implementação de programas de educação postural nas escolas demonstra obter benefícios para as crianças, obtendo ganhos em saúde, com os enfermeiros especialistas em enfermagem de reabilitação a assumir um papel relevante, pelo que se justifica o investimento nesta temática.

\section{REFERÊNCIAS BIBLIOGRÁFICAS}

1. Zapater AR, Silveira DM, Vitta A de, Padovani CR, Silva JCP da. Postura sentada: a eficácia de um programa de educação para escolares. Cien Saude Colet. 2004;9(1):191-9.

2. Santos CIS, Cunha ABN, Braga VP, Saad IAB, Ribeiro M, Conti PBM, et al. Ocorrência de desvios posturais em escolares do ensino público fundamental de Jaguariúna, São Paulo. Rev Paul Pediatr. 2009;27(1):74-80.

3. Schiaffino AN. Avaliação de desvios posturais em crianças entre 11 e 15 anos do Porto. 2010;2-59.

4. Rocha BMP da. Contributos para o plano nacional de saúde 20112016. Assoc Port dos Enfermeiros Reabil. 2010;1-5.

5. Costa R, Rafael M, Silva C, Castilho C, Corrêa P, Galvan T, et al. Patologias relacionadas à má postura em ambiente escolar revisão de literatura. Rev Perspect Ciência e Saúde [Internet]. 2018;3(2):79-89.

6. Carvalho GS de, Ferreira A, Tracana RB. Prevenção de más posturas corporais em crianças dos 7 aos 12 anos, do litoral e do interior de Portugal. Atas do VIII SIEFLAS (Seminário Int ; Lazer e Saúde) S Luís; Univ Fed do Maranhão (UFMA); Bras DVD (ISBN 978989-8537-00-3; 978-989-; 8537-01-0). 2013;1-15.

7. Santos M do CP. Caixa da Prevenção: Comportamento Postural, Dor e Qualidade de Vida em Jovens do $2^{\circ}$ Ciclo. Escola Superior deTecnologia da Saúde de Coimbra; 2017.

8. Milbradt SN, Pranke GI. Aspectos da coluna vertebral relacionados à postura em crianças e adolescentes em idade escolar. Fisioter Bras. 2011;12(2):127.

9. Santos SG dos. Educação postural mediante um trabalho teórico. Rev Bras Atividade Física Saúde. 1998;3(2):32-42.

10. Knoplich J. Viva bem com a coluna que você tem: dores nas costas - tratamento e prevenção. 31 ed. São Paulo: Ibrasa; 2005.

11. Ribeiro AFM, Ribeiro JDO, Tomasini FB, Fulber AS, Munaretto CM, Sousa $\mathrm{AH}$, et al. Postura corporal em escolares: uma revisão da literatura. Ciência em Mov Mov Reabil e Saúde [Internet]. 2017;19(38):17-25.

12. Candotti CT, Macedo $\mathrm{CH}$, Noll M, Freitas $\mathrm{K}$ De. Escola de postura: uma metodologia adaptada aos pubescentes. Rev Mackenzie Educ Física e Esporte. 2010;9(2):91-100.

13. Matos M, Barreiras C, Festas C. Peso máximo da mochila recomendado para crianças (6-12 anos) em contexto escolar: Protocolo de scoping review. Revista Portuguesa de Enfermagem de Reabilitação. 2019;2:49-52.

14. Coury HJC. Self-administered preventive programme for sedentary workers: reducing musculoskeletal symptoms or increasing awareness? Appl Ergon. 1998;29(6):415-21.

15. Santos NB, Sedrez JA, Candotti CTCT, Vieira A, Santos NB Dos Sedrez JA, et al. Immediate and follow-up effects of a posture education program for elementary school students. Rev Paul Pediatr. 2017;35(2):199-206.

16. Fernandes CS, Martins T, Rocha NS, Mendes M. “Posture play": A nursing intervention program for children. Aquichan. 2017;17(2):195-203.

17. Kasten AP, Rosa BN, Schmit EFD, Noll M, Candotti C. Prevalência de desvios posturais na coluna em escolares: revisão sistemática com metanálise. J Desenvolv Hum. 2017;27(1):99-108. 
18. Brzek A, Plinta R. Exemplification of Movement Patterns and Their Influence on Body Posture in Younger School-Age Children on the Basis of an Authorial Program "I Take Care of My Spine". Medicine (Baltimore) [Internet]. 2016 Mar;95(9):e2855-e2855.

19. Dullien S, Grifka J, Jansen P. Cluster-randomized, controlled evaluation of a teacher led multi factorial school based back education program for 10 to 12 -year old children. BMC Pediatr [Internet]. 2018;18(1):312.

20. Vieira Batistão $M$, Carnaz L, de Fátima Carreira Moreira R, de Oliveira Sato T. Effects of a muscular stretching and strengthening school-based exercise program on posture, trunk mobility, and musculoskeletal pain among elementary schoolchildren - a randomized controlled trial. Fisioter em Mov [Internet]. 2019 Jan;32(1):1-13.

21. Direção-Geral da Saúde. Programa Nacional de Saúde Escolar. Lisboa; 2015.

22. Direção Geral da Saúde. Plano de ação para a literacia em saúde Portugal 2019-2021. 2019;6. 